\title{
Surfactant proteins in pulmonary alveolar proteinosis in adults
}

\author{
F. Brasch*,\#, J. Birzele*, M. Ochs”, S.H. Guttentag ${ }^{\oplus}$, O.D. Schoch ${ }^{+}$, A. Boehler ${ }^{\S}$, M.F. Beers ${ }^{f}$, \\ K.M. Müller*, S. Hawgood**, G. Johnen ${ }^{\# \# \#}$
}

Surfactant proteins in pulmonary alveolar proteinosis in adults. F. Brasch, J. Birzele, M. Ochs, S.H. Guttentag, O.D. Schoch, A. Boehler, M.F. Beers, K.M. Müller, S. Hawgood, G. Johnen. C) ERS Journals Ltd 2004.

ABSTRACT: Pulmonary alveolar proteinosis (PAP) is a rare disorder characterised histologically by an intra-alveolar accumulation of fine granular eosinophilic and periodic acid-Schiff positive material.

In a retrospective study, the composition of the intra-alveolarly accumulated material of adult patients with PAP was analysed by means of immunohistochemistry and Western blotting.

In patients with PAP, the current authors found an intra-alveolar accumulation of surfactant protein (SP)-A, precursors of SP-B, SP-B, variable amounts of mono-, di-, and oligomeric SP-C forms, as well as SP-D. Only in one patient was a precursor of SP-C detected. By means of immuno-electron microscopy, the current authors identified not only transport vesicles labelled for precursors of SP-B and SP-C, but also transport vesicles containing either precursors of SP-B or SP-C in type-II pneumocytes in normal human lungs.

It is concluded that pulmonary alveolar proteinosis in adults is characterised by an intra-alveolar accumulation of surfactant protein A, precursors of surfactant protein B, and surfactant proteins $\mathrm{B}, \mathrm{C}$ and $\mathrm{D}$. The current data provide evidence that not only an impairment of surfactant clearance by alveolar macrophages, but also an abnormal secretion of transport vesicles containing precursors of surfactant protein B (but not surfactant protein $C$ ) and an insufficient palmitoylation of surfactant protein $C$, which may lead to the formation of di- and oligomeric surfactant protein $\mathrm{C}$ forms, play a role in the pathogenesis of pulmonary alveolar proteinosis.

Eur Respir J 2004; 24: 426-435.

\begin{abstract}
*Institute of Pathology, University Hospital "Bergmannsheil", and \#\#Institute of Occupational Medicine, Ruhr University, Bochum, and ${ }^{\#}$ Division of Electron Microscopy, Dept of Anatomy, University of Göttingen, Göttingen, Germany. "Division of Neonatology, Dept of Paediatrics, and ${ }^{f}$ Pulmonary and Critical Care Division, Dept of Medicine, University of Pennsylvania School of Medicine, Philadelphia, PA, and **Dept of Paediatrics and Cardiovascular Research Institute, University of California San Francisco, San Francisco, CA, USA. ${ }^{+}$Division of Pulmonary Medicine, Cantonal Hospital, St. Gallen, and ${ }^{\S}$ Division of Pulmonary Medicine and Lung Transplant Program, University Hospital, Zürich, Switzerland.
\end{abstract}

Correspondence: F. Brasch, Institute of Pathology, University Hospital "Bergmannsheil", Buerkle-de-la-Camp Platz 1, D-44789 Bochum, Germany.

Fax: 492343026671

E-mail: Frank.E.Brasch@ruhr-uni-bochum.de

Keywords: Precursors of surfactant protein B, pulmonary alveolar proteinosis, surfactant

Received: July 32003

Accepted after revision: May 32004

This study was partially supported by NIH P01-19737 (M.F. Beers) and NIH HL-59959 (S.H. Guttentag).
Pulmonary alveolar proteinosis (PAP) was first described in 1958 by ROSEN et al. [1] as a unique lung disorder characterised histologically by an intra-alveolar accumulation of fine granular eosinophilic and periodic acid-Schiff (PAS) positive material. PAP represents a heterogeneous group of congenital, idiopathic or secondary diseases in newborns, infants and adults. Idiopathic PAP in adults is the most common form. Recently, a neutralising antibody against granulocyte macrophage-colony stimulating factor (GM-CSF) in adult patients with idiopathic PAP has been characterised, and it has been shown that such autoantibodies are diagnostic for idiopathic PAP $[2,3]$. The surfactant accumulation in GM-CSF-deficient mice could be corrected by the application of GM-CSF. Also, the selective expression of GM-CSF in pulmonary epithelial cells and administration of GM-CSF shows therapeutic activity in patients with idiopathic PAP $[4,5]$. Secondary alveolar proteinosis has been reported in several clinical settings; it comprises aberrant responses to infection and inhalation of minerals or chemicals, or it might be associated with underlying diseases, such as lymphomas, and acute and chronic leukaemia [6].

Although the intra-alveolar accumulation of surfactant lipids, surfactant protein (SP)-A, SP-B and SP-D has been well-established in adult patients with idiopathic PAP [6], little is known about SP-C, and precursors of SP-B and SP-C. In a retrospective study, the composition of the intra-alveolar accumulated material of adult patients with idiopathic and secondary PAP was analysed by means of immunohistochemistry and Western blotting.

\section{Materials and methods}

\section{Patients with pulmonary alveolar proteinosis}

In 26 adult patients, alveolar proteinosis was diagnosed on the basis of symptoms, computed tomography scans, and either transbronchial (21 patients) or open lung biopsies (four patients) or bronchoalveolar lavage (BAL) fluid (one patient) [7, 8]. A pulmonary infection was excluded in all patients. Haematoxylin-eosin and PAS staining was performed according to standard procedures. BAL fluid was available from 12 patients. GM-CSF-neutralising autoantibodies were retrospectively assayed by Western blotting in pre-treatment sera from five patients with idiopathic PAP by K. Nakata (Dept of Respiratory Diseases, Research Institute, International 
Medical Centre of Japan, Tokyo, Japan) [3]. Two of these patients were included in the previously performed GM-CSF treatment study [5]. The baseline characteristics of patients with PAP are summarised in table 1.

\section{Normal human lungs}

As controls for immunohistochemistry and immuno-electron microscopy (EM), eight nontransplanted human single donor lungs and lung specimens from 10 autopsy cases (patients died of acute cardiac failure and had no history of pulmonary disease) were used. Donor lungs were used for investigation only if they could not be made available for a suitable recipient by the Eurotransplant Foundation Centre, Leiden, The Netherlands. All lungs were carefully examined by two board certified pathologists (F. Brasch, K.M. Müller). None of the nontransplanted donor lungs and lung specimens from autopsies used for the current study showed any pathological changes. Fixation of human lungs was performed by instillation of the fixative via the airways to ensure rapid and uniform fixation.

\section{Human type-II pneumocytes}

Isolated type-II pneumocytes were prepared as previously described [9]. Briefly, $1-\mathrm{mm}^{3}$ tissue explants of human foetal lung parenchyma from second trimester therapeutic abortions were cultured overnight in Waymouth's media (protocols were approved by the Committee for Human Research, Children's Hospital of Philadelphia, PA, USA). Following overnight culture, $10 \mathrm{nM}$ dexamethasone, $0.1 \mathrm{mM} 8-\mathrm{Br}-$ cAMP and $0.1 \mathrm{mM}$ isobutylmethylxanthine were added to the medium and explants were cultured for 6-7 days to induce type-II cell differentiation. Type-II pneumocytes were isolated from tissue explants by enzymatic digestion and panning on plastic culture dishes to remove fibroblasts.

\section{Antisera}

The monoclonal antibody PE-10 against SP-A was purchased from Dako (Glostrup, Denmark). Rabbit antiserum against mature SP-B was purchased from Chemicon (Temecula, CA, USA). A monoclonal antibody against human SP-B was kindly donated by Y. Suzuki (Dept of Ultrastructural Research, Kyoto University, Japan). Polyclonal antiserum against SP-D was kindly given by E.C. Crouch (Dept of Pathology and Immunology, Barnes-Jewish Hospital, St Louis, MO, USA). Polyclonal rabbit antisera against SP-B proprotein (anti-proSPB) were generated using the recombinant C-terminal propeptide. The polyclonal antiserum against the proSP-C epitopes $\mathrm{E}^{11}-\mathrm{R}^{23}$ (anti-proSP-C) was characterised previously [10, 11]. A polyclonal antiserum against mature recombinant human SP-C was kindly provided by W. Steinhilber (Altana Pharma, Konstanz, Germany).

\section{Immunohistochemistry}

Tissue preparation and immunostaining of lung specimens from patients with PAP, nontransplanted human single donor lungs and autopsy cases were performed as previously described in detail $[12,13]$. Briefly, immunostaining of dewaxed paraffin sections was performed in an automated staining system according to the specifications of the manufacturer (TechMate 500; Dako). The immunoreaction was demonstrated using an alkaline phosphatase-anti-alkaline phosphatase kit (Dako). Fast Red (Dako) was used as alkaline-phosphatase substrate.

\section{Statistical analysis}

The staining of intra-alveolar material was graded according to the following system: grade 0 , no staining within the alveoli; grade 1, only a focal staining within the alveoli; grade 2 , more than five alveoli are completely filled by a moderately

Table 1.-Baseline characteristics of the patients with pulmonary alveolar proteinosis (PAP)

\begin{tabular}{|c|c|c|c|c|}
\hline Patient no. & Sex & Age at onset of symptoms yrs & Material & Diagnosis, underlying disease \\
\hline 1 & M & 20 & TBB, BAL, serum & Idiopathic PAP, GM-CSF autoantibody \\
\hline 2 & $\mathrm{~F}$ & 18 & TBB, BAL, serum & Idiopathic PAP, GM-CSF autoantibody \\
\hline 3 & M & 32 & BAL, serum & Idiopathic PAP, GM-CSF autoantibody \\
\hline 4 & $\mathrm{~F}$ & 35 & Open lung biopsy, BAL, serum & Idiopathic PAP, GM-CSF autoantibody \\
\hline 5 & $\mathrm{~F}$ & 34 & TBB, BAL & Idiopathic PAP \\
\hline 6 & M & 45 & TBB, BAL & Idiopathic PAP \\
\hline 7 & M & 35 & Open lung biopsy, BAL & Idiopathic PAP \\
\hline 8 & M & 42 & TBB, BAL & Idiopathic PAP \\
\hline 9 & $\mathrm{~F}$ & 44 & TBB, BAL & Idiopathic PAP \\
\hline 10 & M & 31 & TBB, BAL & Idiopathic PAP \\
\hline 11 & M & 24 & TBB, BAL & Secondary PAP, acute silicoproteinosis \\
\hline 12 & $\mathrm{~F}$ & 51 & TBB, BAL & Idiopathic PAP \\
\hline 13 & $\mathrm{~F}$ & 57 & TBB & Idiopathic PAP \\
\hline 14 & $\mathrm{M}$ & 47 & TBB & Idiopathic PAP \\
\hline 15 & $\mathrm{M}$ & 34 & TBB & Idiopathic PAP \\
\hline 16 & $\mathrm{M}$ & 52 & TBB & Idiopathic PAP \\
\hline 17 & $\mathrm{M}$ & 34 & Open lung biopsy & Idiopathic PAP \\
\hline 18 & $\mathrm{~F}$ & 54 & TBB & Idiopathic PAP \\
\hline 19 & $\mathrm{~F}$ & 29 & TBB & Idiopathic PAP \\
\hline 20 & $\mathrm{~F}$ & 40 & TBB & Idiopathic PAP \\
\hline 21 & $\mathrm{~F}$ & 34 & TBB & Idiopathic PAP \\
\hline 22 & M & 38 & Open lung biopsy & Idiopathic PAP \\
\hline 23 & $\mathrm{~F}$ & 29 & TBB & Idiopathic PAP \\
\hline 24 & $\mathrm{M}$ & 60 & TBB & Secondary PAP, myelodysplastic syndrome \\
\hline 25 & $\mathrm{M}$ & 30 & TBB, serum & Idiopathic PAP, GM-CSF autoantibody \\
\hline 26 & $\mathrm{~F}$ & 42 & TBB & Idiopathic PAP \\
\hline
\end{tabular}

M: male; F: female; TBB: transbronchial biopsy; BAL: bronchoalveolar lavage fluid; GM-CSF: granulocyte macrophage-colony stimulating factor. 
stained material; and grade 3, more than five alveoli are completely filled by a strongly stained material. All sections were blinded by a technician and independently graded by three investigators (F. Brasch, J. Birzele, and K.M. Müller). Differences between controls and PAP were analysed using the Mann-Whitney rank sum test.

\section{Immuno-electron microscopy}

Tissue preparation and immunogold labelling of normal human lungs were performed as described in detail elsewhere $[12,13]$. Briefly, ultrathin sections from cryosubstituted and Lowicryl HM20-embedded(Polysciences, Eppelheim, Germany) lung specimens were triple-labelled for proSP-B, proSP-C and SP-B, according to the following procedure. First, sections were labelled with the primary polyclonal antiserum against proSP-B and the monoclonal antibody against SP-B without having been mounted on a grid. The immunoreaction was visualised by incubation with a secondary 10-nm anti-rabbit and 15-nm anti-mouse gold-coupled antibody. Finally, sections were mounted with the labelled plane downwards on a Formvar-coated copper or nickel grid and dried overnight. The next day, the grids were labelled with the third primary antibody against proSP-C and a secondary 5-nm anti-rabbit gold-coupled antibody. Labelled sections were systematically viewed and photographed with a Leo EM 900 electron microscope (Leo, Oberkochen, Germany) at $50 \mathrm{kV}$.

\section{Detection of surfactant proteins by Western blot analysis}

For immunoblotting, alveolar proteinosis fluids or extracts of type-II pneumocytes were separated on 4-12\% NuPage
Bis-Tris polyacrylamide gels and then transferred to nitrocellulose membranes according to the manufacturer's protocol (Invitrogen, Carlsbad, CA, USA). After blocking ( $2 \mathrm{~h}$ in $5 \%$ foetal calf serum $/ 0.1 \%$ Tween $20 / 0.5 \%$ bovine serum albumin in tris-buffered saline $\mathrm{pH}$ 7.5), membranes were incubated overnight with antibodies against proSP-B, SP-B or proSP-C. An anti-rabbit immunoglobulin $G$ alkaline-phosphatase conjugate (substrate: nitrobluetetrazolium/5-bromo-4-chloro3-indolylphosphate) was used as the secondary antibody (Promega, Madison, WI, USA). For the detection of precursors of SP-B, SP-B and precursors of SP-C, Western blots were performed under nonreducing conditions. Since SP-C forms amyloid fibrils in patients with PAP that could be resolved by sodiumdodecylsulphate-polyacrylamide gel electrophoresis only under reducing conditions, immunoblots for SP-C were performed under nonreducing and reducing conditions (fig. 1).

\section{Results}

\section{Distribution of surfactant proteins in normal human lungs}

As previously described [13], SP-A showed a weak labelling of type-II pneumocytes and a focal positive staining at the alveolar epithelial surface (grade 1; fig. 2a). Type-II pneumocytes were weakly stained for precursors of SP-B (fig. 3a), but showed a strong granular staining for mature SP-B (fig. 4a). In the alveolar space, SP-B (grade 1; fig. 4a) was found, but no precursors of SP-B (grade 0; fig. 3a), as described previously in detail [14]. The immunohistochemical staining of proSP-C is restricted to type-II pneumocytes in human lungs (fig. 5a) as previously shown [12]. Only a very weak staining of type-II pneumocytes for SP-D was observed and,
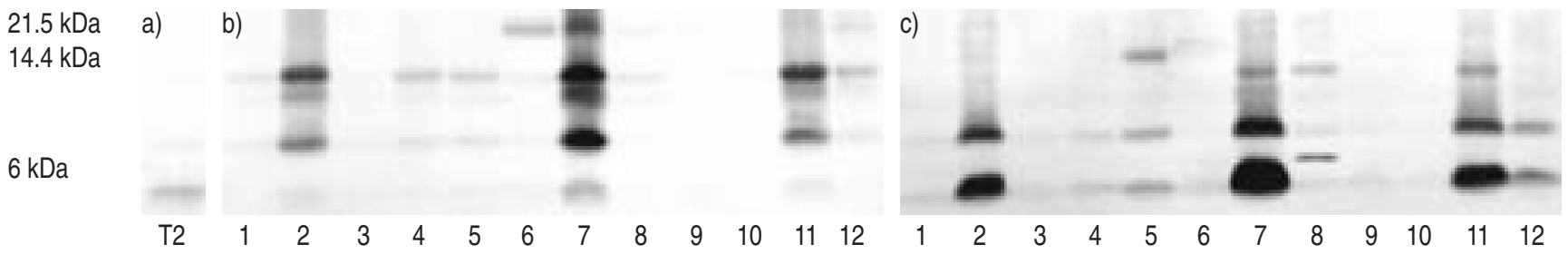

Fig. 1.-Electrophoretic analysis of surfactant protein (SP)-C forms in bronchoalveolar lavage fluids of patients with pulmonary alveolar proteinosis. b) In alveolar proteinosis fluids, anti-SP-C identified, under nonreducing conditions, monomeric $\sim 4 \mathrm{kDa}$, dimeric $\sim 8 \mathrm{kDa}$, oligomeric $\sim 12 \mathrm{kDa}$ and $\sim 16 \mathrm{kDa}$ SP-C forms. The amount of monomeric, dimeric and oligomeric SP-C forms markedly varied. Whilst under nonreducing conditions only small amounts of monomeric SP-C were detectable, it markedly increased under reducing conditions and the oligomeric SP-C forms decreased or disappeared (c). Furthermore, in one patient an additional aberrant $\sim 5 \mathrm{kDa}$ SP-C form was identified under reducing conditions (c). In isolated human type-II pneumocytes (T2; a) only monomeric $\sim 4 \mathrm{kDa}$ SP-C was present.
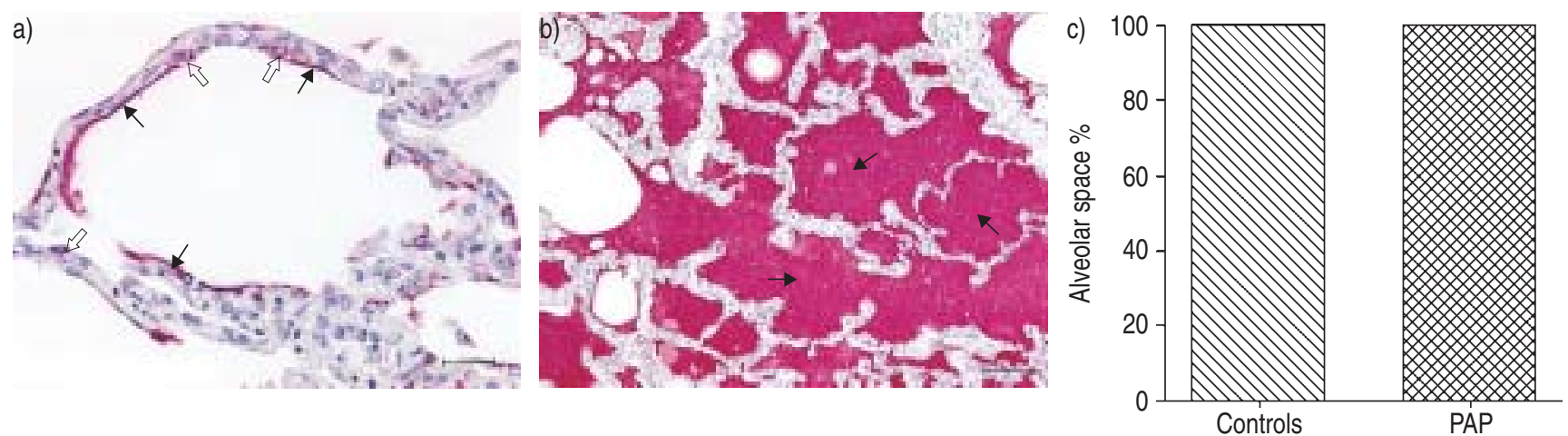

Fig. 2. - Distribution of the surfactant protein (SP)-A in controls (a) and pulmonary alveolar proteinosis (PAP) (b). As previously described in detail [13], type-II pneumocytes (open arrows) are weakly labelled for SP-A (a). A positive staining is also present on the alveolar surface (arrows; grade 1: $\mathbb{Q}$; a and c). In patients with PAP, the intra-alveolarly accumulated surfactant (arrows) shows a strong staining (grade 3 : for SP-A (b and c). Scale bars $=25$ and $100 \mu \mathrm{m}$; for $\mathrm{a}$ and $\mathrm{b}$, respectively. 

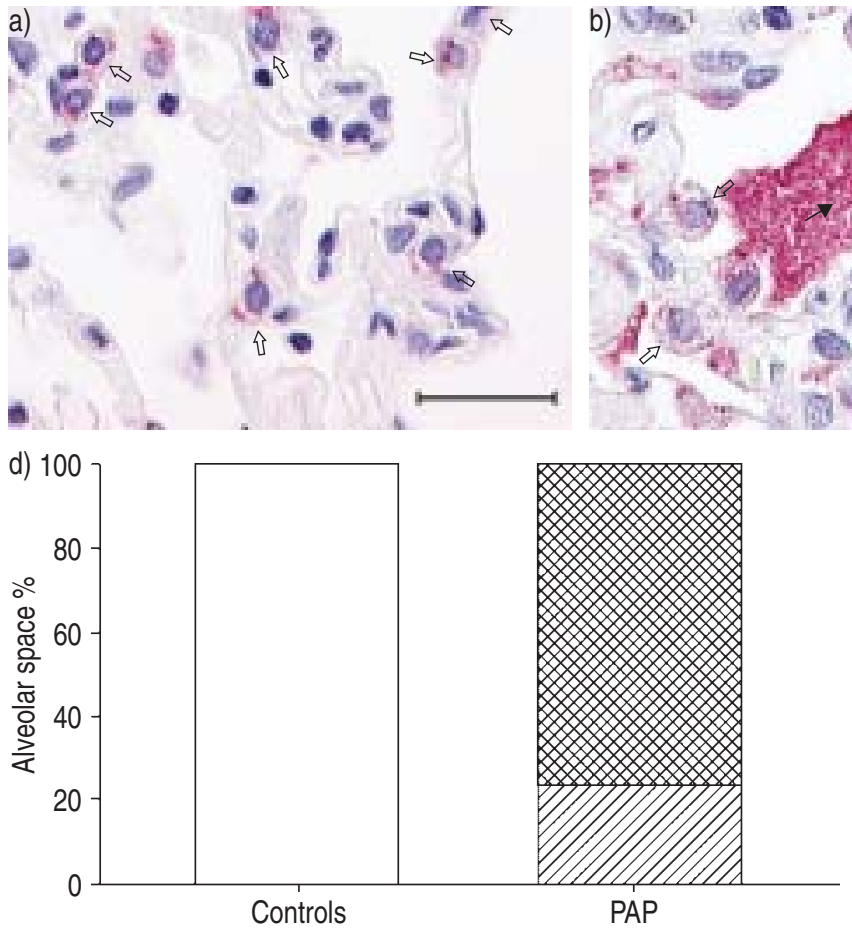
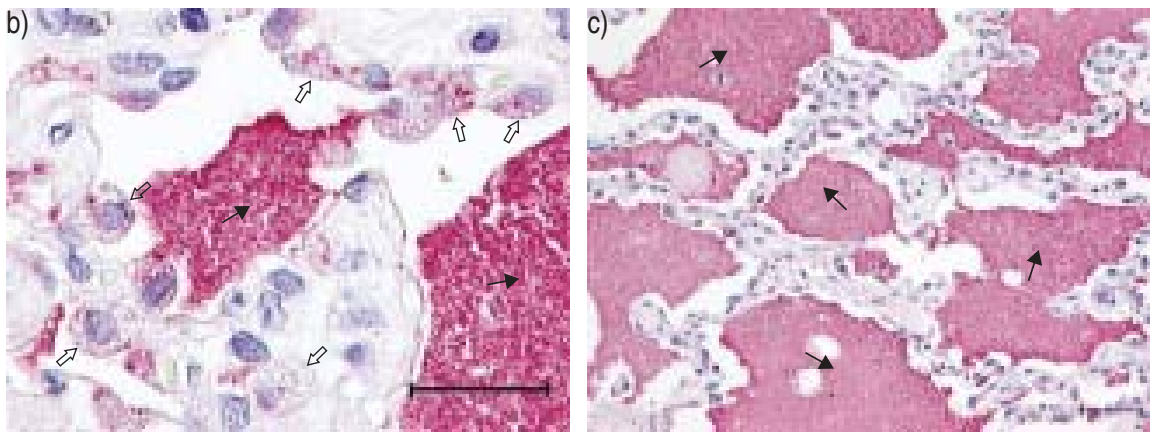

e)

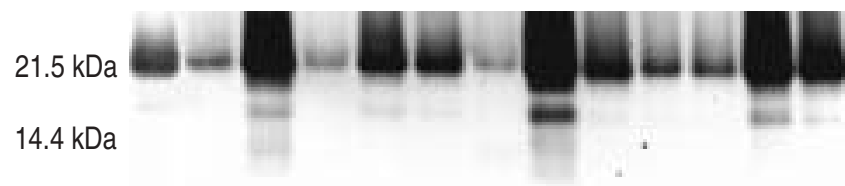

$6 \mathrm{kDa}$

Fig. 3.-Distribution of precursors of surfactant protein (SP)-B in controls (a) and pulmonary alveolar proteinosis (PAP) (b and c). While type-II pneumocytes (open arrows) are weakly stained for proSP-B, no precursors of SP-B were identified in the alveolar space in controls (grade 0; a). Corresponding to the moderate-to-strong staining (grade 2-3) of the intra-alveolarly accumulated material (c; arrows) for precursors of SP-B in patients with PAP (b-d; grade 0: $\square$, grade 2: $\mathscr{Z}$, grade 3: $\mathbf{0}$ ), anti-proSP-B identified $\sim 23 \mathrm{kDa}$ and $\sim 17 \mathrm{kDa}$ precursors of SP-B in alveolar proteinosis fluids (e, 1-12: bronchoalveolar lavage fluids from patients with PAP). Despite an intra-alveolar accumulation of precursors of SP-B, only a weak cytoplasmic staining of type-II pneumocytes (open arrows) was observed, while the lamellar bodies were negative (b). Precursors of SP-B identified in PAP were also found in isolated human type II-pneumocytes (T2) (e). Scale bars=25 (a and b) and $50 \mu \mathrm{m}$ (c).

only focally, a positively stained material in the alveoli (grade 1; fig. 6a) was detected.

To characterise intracellular compartments containing precursors of SP-B, SP-B and precursors of SP-C in normal human lungs, an immuno-EM triple-labelling was performed. Golgi vesicles were found to contain either precursors of SP-B (10-nm gold particles) or SP-C (5-nm gold particles) (fig. 7a). Golgi vesicles did not contain SP-B (15-nm gold particles), nor were there Golgi vesicles with both precursors of SP-B and SP-C. Two types of multivesicular bodies (mvb) were detected and defined as type-A or -B mvb; type-A mvb contained both precursors of SP-B and mature SP-B, but no precursors of SP-C (fig. 7a). Type-B mvb contained precursors of SP-B and SP-C, sometimes with mature SP-B in addition (fig. 7b). Composite and lamellar bodies stained for SP-B (15-nm gold particles; fig. 7a and b). Furthermore, precursors of SP-C were also identified in composite bodies and in a few lamellar bodies as described previously [12].

\section{Electrophoretic analysis of human type-II pneumocytes}

In type-II pneumocytes, anti-proSP-B (fig. 3e) identified two precursors of SP-B $(\sim 23 \mathrm{kDa}$ and $\sim 17 \mathrm{kDa})$. Furthermore, type-II pneumocytes contained mature monomeric $\sim 8 \mathrm{kDa}$ and dimeric $\sim 18 \mathrm{kDa}$ SP-B (fig. $4 \mathrm{~d}$ ). Anti-proSP-C identified $\sim 21 \mathrm{kDa}, \sim 14 \mathrm{kDa}$ and $\sim 10 \mathrm{kDa}$ precursors of SPC (fig. 5d) and anti SP-C detected monomeric SP-C in type-II pneumocytes (fig. 1).

\section{Distribution of surfactant proteins in pulmonary alveolar proteinosis}

In all patients with PAP, more than five alveoli were completely filled by a material that showed a strong staining for SP-A (grade 3; fig. 2b and c) and a moderate-to-strong staining for SP-D (grade 2-3; fig. $6 \mathrm{~b}$ and c). In contrast to normal human lungs (fig. 3a), the intra-alveolar accumulated material in PAP showed a moderate-to-strong staining for precursors of SP-B (grade 2-3; fig. 2b-d), whilst the staining pattern of type-II pneumocytes (fig. 3b) was similar to normal human lungs (fig. 3a). Corresponding to the moderate-tostrong staining of the intra-alveolar material for precursors of SP-B, anti-proSP-B identified a $\sim 23 \mathrm{kDa}$ precursor of SP-B in BAL fluids of all PAP patients (fig. 2e; patients 1-12). Furthermore, a $\sim 17 \mathrm{kDa}$ precursor of SP-B was detectable in BAL fluids of four patients (fig. 2e; patients 2, 4, 7, and 11).

Anti-SP-B showed a moderate-to-strong staining (grade 2-3) of the intra-alveolar accumulated material (fig. $4 \mathrm{~b}$ and c). Dimeric $\sim 18 \mathrm{kDa}$ and variable amounts of monomeric $\sim 8 \mathrm{kDa}$ SP-B were present in the BAL fluids of all patients (fig. 4d; patients 1-12). Statistical analysis of the immunohistochemical staining pattern of the intra-alveolar material for SP-A, precursors of SP-B (proSP-B), mature SP-B and SP-D showed a significant difference between controls and PAP $(\mathrm{p}<0.0001)$.

In all cases, anti-proSP-C moderately stained type-II pneumocytes (fig. 5b). Only in one case was a staining of the intra-alveolar material (grade 2) observed (not shown) and a $\sim 12 \mathrm{kDa}$ precursor of SP-C was detectable in BAL fluid (fig. 5d; patient 8). However, the difference among the staining pattern of controls and PAP was not great enough to exclude the possibility that the difference was due to random sampling variability.

Variable amounts of monomeric $\sim 4 \mathrm{kDa}$, dimeric $\sim 8 \mathrm{kDa}$, oligomeric $\sim 12 \mathrm{kDa}$ and, in addition, $\sim 16 \mathrm{kDa}$ SP-C forms were found in BAL fluids of patients with PAP under nonreducing (fig. 1b) as well as reducing conditions (fig. 1c). Only in patient 8 was an aberrant $\sim 5 \mathrm{kDa}$ SP-C detectable (fig. 1b). 

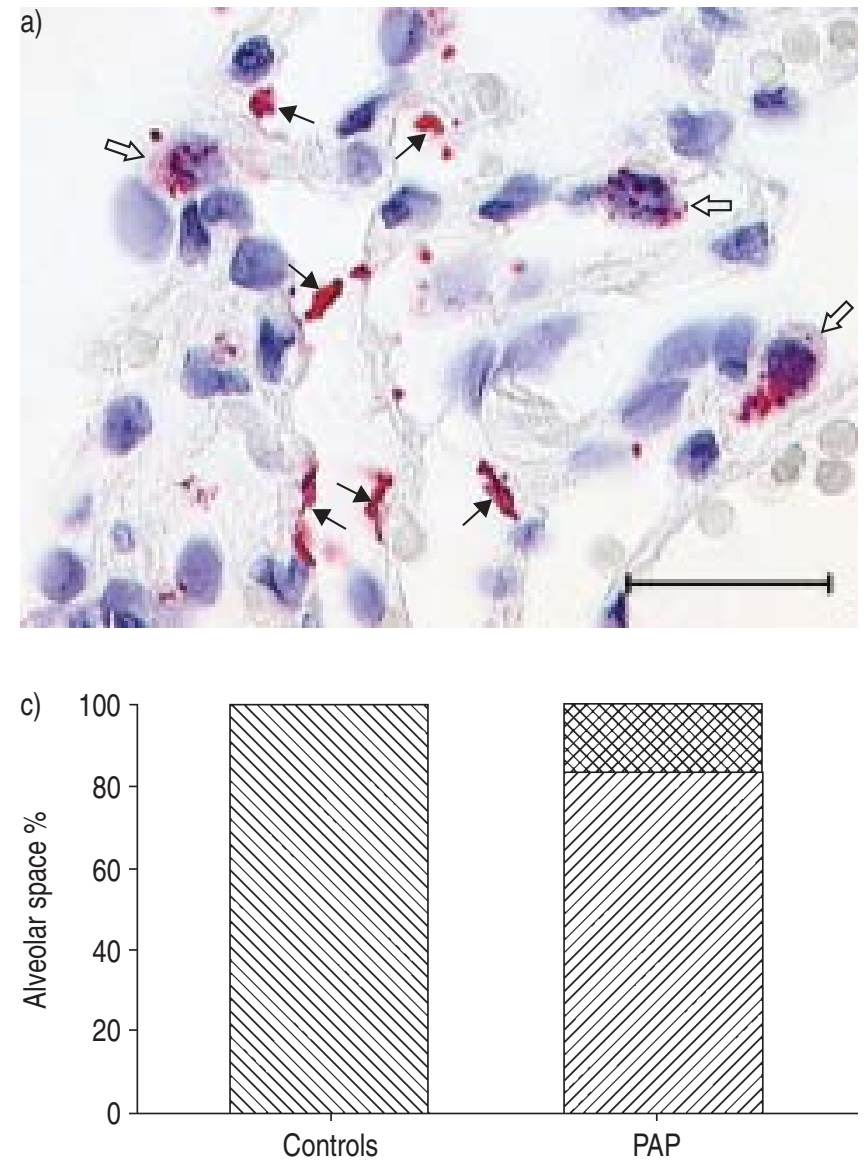

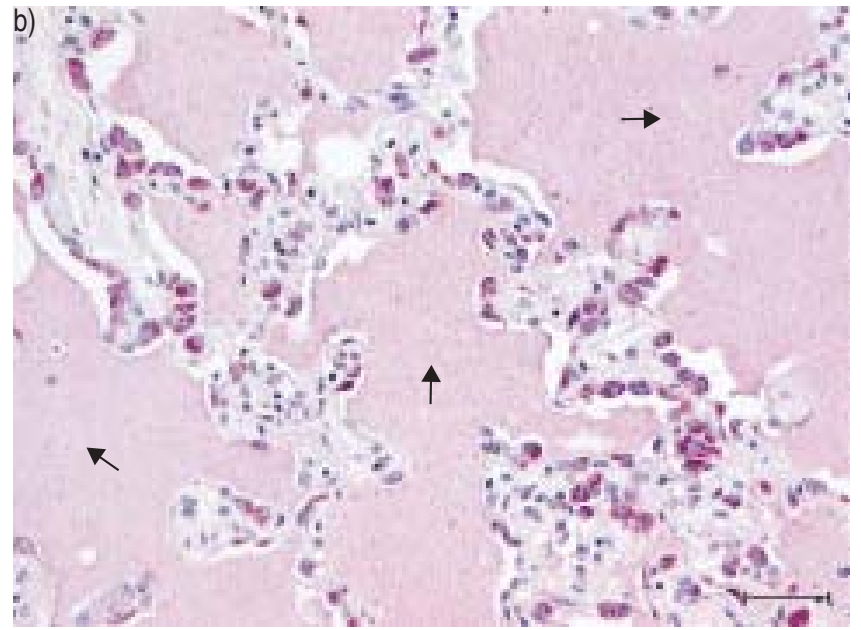

d)

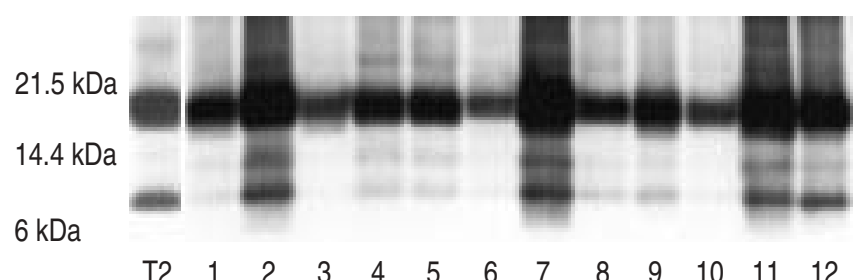

Fig. 4. - Distribution of surfactant protein (SP)-B in controls (a) and pulmonary alveolar proteinosis (PAP) (b). In line with the ultrastructural localisation of SP-B mainly in lamellar bodies, immunohistochemistry on type-II pneumocytes (open arrows) showed a moderate to strong granular staining pattern in controls and patients with PAP (a and b). In line with the ultrastructural localisation of SP-B in intra-alveolar lamellar body-like surfactant forms and tubular myelin figures in the alveolar space, only a focal immunohistochemical staining (arrows, grade 1) was found in controls (a). In line with the identification of monomeric $\sim 8 \mathrm{kDa}$ and dimeric $\sim 18 \mathrm{kDa}$ SP-B in alveolar proteinosis fluids (1-12: bronchoalveolar lavage fluids from patients with PAP; d), the intra-alveolarly accumulated material in patients with PAP (arrows) showed a moderate-to-strong staining (grade 2-3) for SP-B (b and c; grade 1: $\mathbb{Q}$, grade 2: $\mathbb{Z}$, grade 3: $\mathbf{D}$ ). Similar to the immunohistochemical staining pattern in healthy humans (fig. 5), type-II pneumocytes (open arrows) showed a strong granular staining pattern for SP-B (d). Monomeric and dimeric SP-B identified in alveolar proteinosis fluids (1-12) were also found in isolated human type-II pneumocytes (T2; d). Scale bars=25 and $50 \mu \mathrm{m}$; for $\mathrm{a}$ and $\mathrm{b}$, respectively.

\section{Discussion}

Recent studies have suggested that GM-CSF signalling plays an important role in surfactant homeostasis. GM-CSFand common $\beta$-chain-deficient mice develop a progressive accumulation of surfactant lipids and, additionally, SP-A, SPB, SP-C and SP-D in the alveolar space, similar to PAP in humans [15]. The pre-treatment sera of five patients with idiopathic PAP were retrospectively assayed by Western blotting for GM-CSF-neutralising autoantibodies [3]. In line with published data [2-4], a neutralising antibody was found in each patient. Two patients were suffering from secondary PAP, either following acute exposure to silica (acute silicoproteinosis) or associated with a myelodysplastic syndrome. Secondary PAP is a rare pulmonary complication after exposure to silica or titanium, or in patients with malignancies of haematopoietic origin, and only a few cases have been described in the literature [6]. Animal models of silica-induced alveolar proteinosis provided evidence that the proteinosis was related to an imbalance between surfactant biosynthesis, secretion and clearance [16]. However, on account of an unaltered expression of SP-A, SP-B, SP-C and SP-D, it has been suggested that an impairment of surfactant clearance by alveolar macrophages, as a result of inhibition of the action of GM-CSF, might underlie PAP in GM-CSF and interleukin (IL)-3/GM-CSF/IL-5 $\beta$ c-receptor-deficient mice [4, 17-19].

The significant differences of the immunohistochemical staining pattern of the intra-alveolar material for SP-A, SP-B and SP-D between controls and patients with PAP correlates well with animal models of PAP and the biochemical findings that SP-A, SP-B and SP-D are increased in alveolar proteinosis lavage fluids in humans [20-23]. However, using immunohistochemistry and Western blotting, a significant intra-alveolar accumulation of precursors of SP-B was also found in all patients with PAP. Since neither the current authors nor others found precursors of SP-B in the alveolar space or alveolar macrophages in normal human lungs [14, 24], the abnormal intra-alveolar appearance of precursors of SP-B in PAP cannot be explained solely by a defective clearance of surfactant components.

SP-B is synthesised as a 381 -amino acid $40-\mathrm{kDa}$ proprotein (proSP-B) in type-II pneumocytes [25]. The processing of the proprotein involves cleavage of the signal peptide, glycosylation of the $\mathrm{C}$-terminus, and cleavage of the $\mathrm{N}$-terminal and C-terminal propeptide [11]. The post-translational processing of proSP-B occurs in type-II pneumocytes between the Golgi complex and mvb prior to transfer of mature SP-B to lamellar 

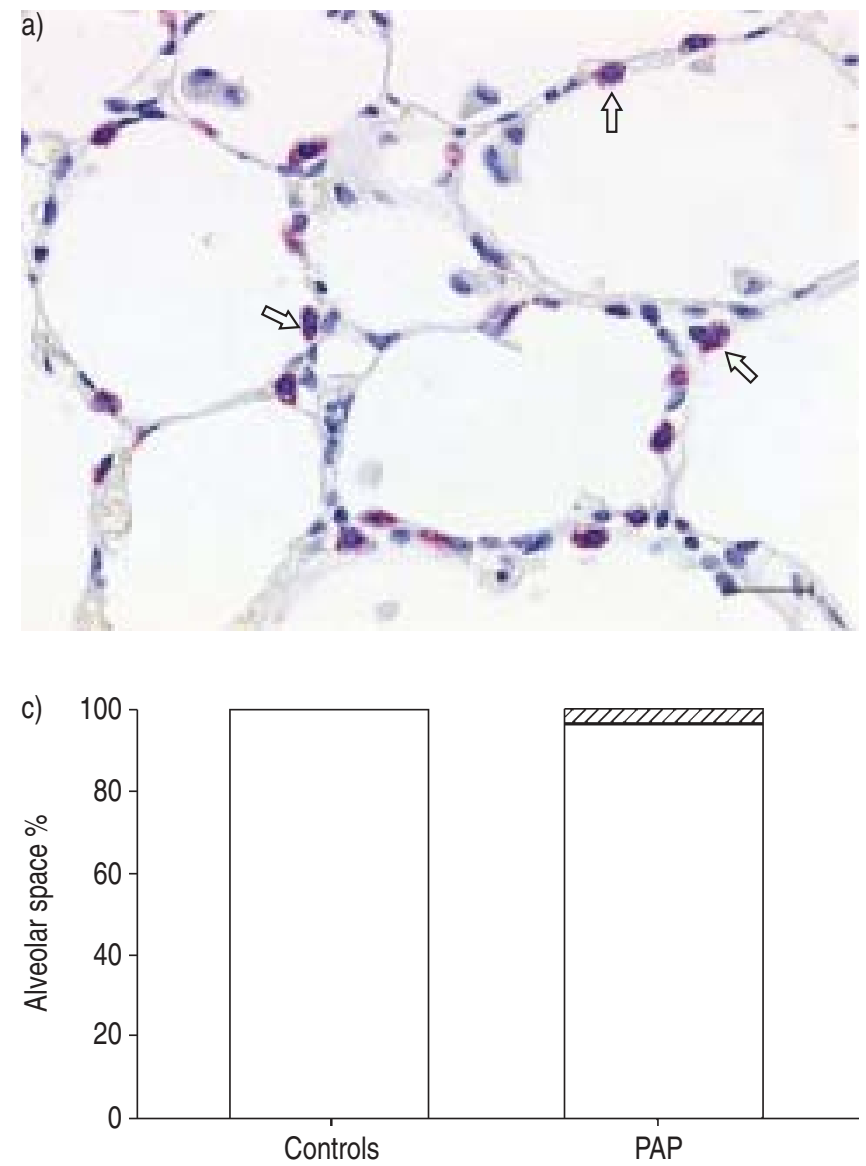

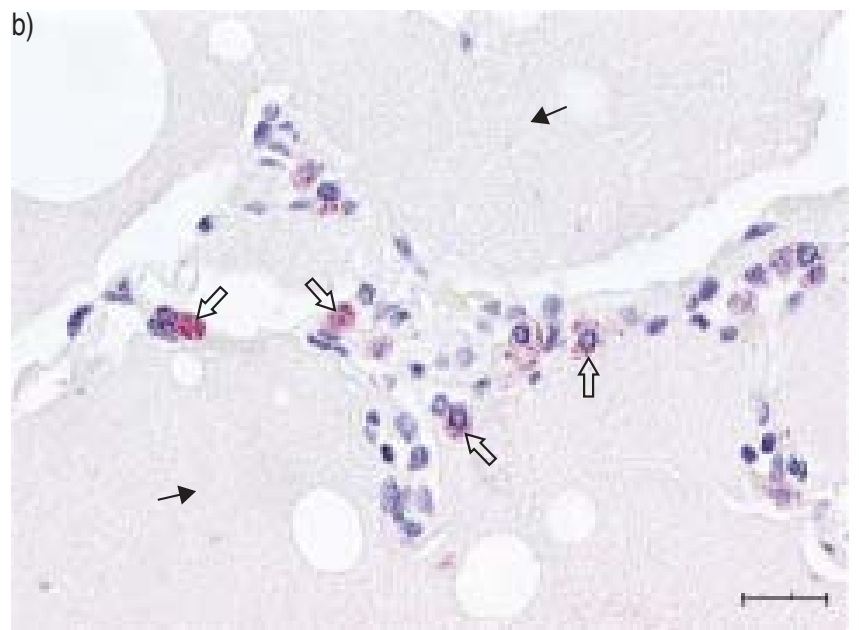

d)

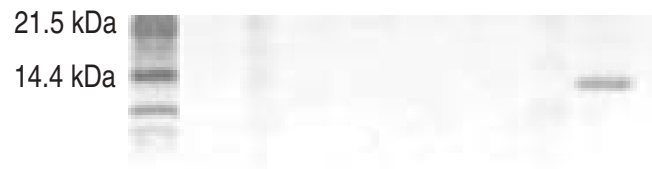

$6 \mathrm{kDa}$

$\begin{array}{lllllllllllll}\mathrm{T} 2 & 1 & 2 & 3 & 4 & 5 & 6 & 7 & 8 & 9 & 10 & 11 & 12\end{array}$

Fig. 5. - Distribution of precursors of surfactant protein (SP)-C in controls (a) and pulmonary alveolar proteinosis (PAP) (b). In line with the electrophoretic analysis (d) of isolated human type-II pneumocytes (T2) and bronchoalveolar lavage fluids of patients with PAP (1-12), type-II pneumocytes (open arrows) showed a cytoplasmic staining pattern for precursors of SP-C in controls and patients with PAP (a and b), whilst the intra-alveolarly accumulated material in PAP (arrows) was negative in most cases (b and c; grade 0: $\square$, grade 2: $\mathbb{Z}$ ). Only in one patient with PAP was a weak-to-moderate staining (grade 2) of the intra-alveolarly accumulated material detected and a $\sim 12 \mathrm{kDa}$ precursor of SP-C in the alveolar proteinosis fluid by Western blotting (c and d; patient 8 ). Scale bars $=25 \mu \mathrm{m}$.
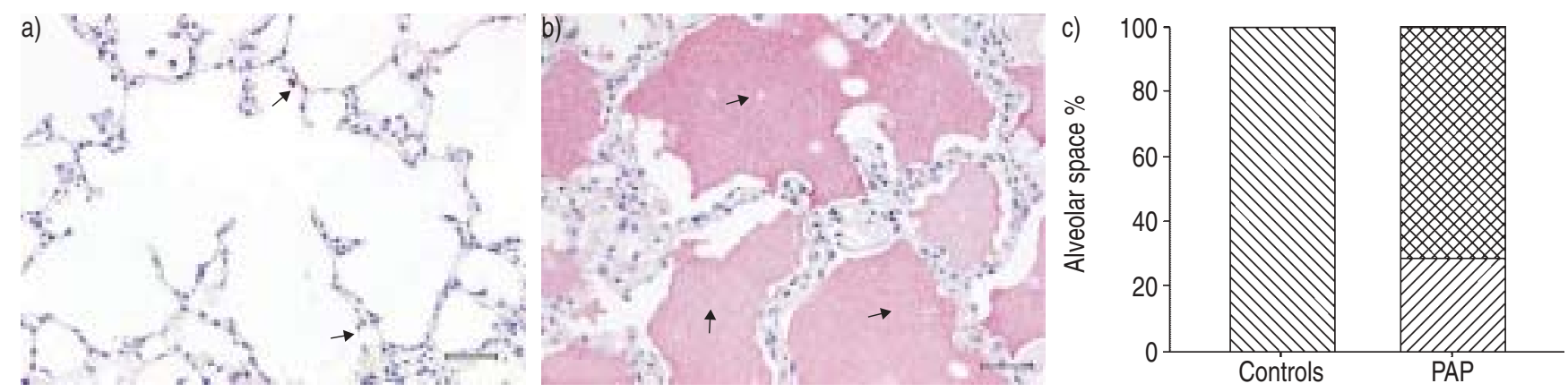

Fig. 6. - Distribution of surfactant protein (SP)-D in controls (a) and patients with pulmonary alveolar proteinosis (PAP) (b). In controls, type-II pneumocytes are very weakly labelled and, in the alveoli, only a focal immunohistochemical staining (grade 1) was found (a and c; grade 1: $\mathbb{Q}$, grade 2: $\mathbb{Z}$, grade 3: $\mathbf{E}$ ). In contrast to controls, the intra-alveolarly accumulated material (arrows) in patients with PAP showed a moderate-tostrong staining (grade 2-3) for SP-D (b and c). Scale bars $=50 \mu \mathrm{m}$.

bodies and secretion in the alveolar space $[11,24]$. In normal human lungs and PAP, type-II pneumocytes showed a moderate-to-strong granular staining pattern for SP-B, consistent with the localisation of SP-B that was mainly in lamellar bodies. Despite the intra-alveolar accumulation of precursors of SP-B in the alveolar space in PAP, anti-proSP-B stained the cytoplasm of type-II pneumocytes similar to normal human lungs, but not the lamellar bodies. Since the precursors identified in alveolar proteinosis fluids that PAP corresponded well in size and antigenic characteristics to the processing intermediates of proSP-B in human type-II pneumocytes, an abnormal enzymatic processing of proSP-B in PAP appears unlikely. Both the staining pattern and biochemical data provide indirect evidence that insufficiently processed proSP-B might be secreted into the alveolar space, bypassing the lamellar bodies. 

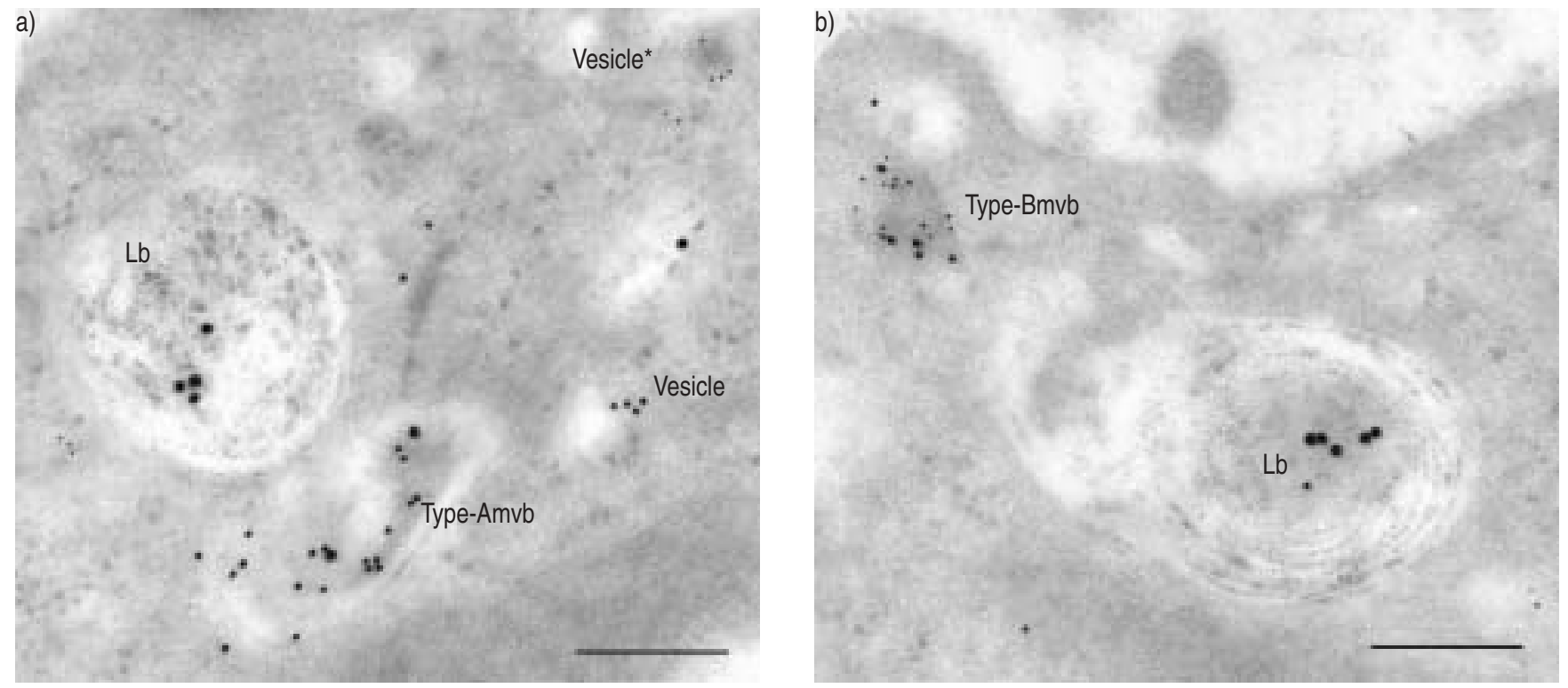

Fig. 7.-Immunolocalisation of precursors of surfactant protein (SP)-B and SP-C in type-II pneumocytes in normal human lungs. Immuno-triple labelling for proSP-B, proSP-C and SP-B identified Golgi vesicles containing either precursors of SP-C (vesicle*) (5-nm gold particles) or SP-B (vesicle) (10-nm gold particles) (a). Furthermore, two types of multivesicular bodies (mvb) were detected: type-A mvb contained both precursors of SP-B and SP-B (15 nm gold particles) (a) type-B mvb contained precursors of SP-B and SP-C and occasionally SP-B (b). Lamellar bodies (lb) primarily stained for SP-B (a and b), with precursors of SP-C seen in a few lb (not shown). Immuno-electron microscopy findings are summarised in figure $8 \mathrm{a}$. Scale bars $=0.25 \mu \mathrm{m}$.

It is generally accepted that, under physiological conditions, both SP-B and SP-C are delivered together by mvb to lamellar bodies and secreted with the lamellar bodies in the alveoli [26, 27]. SP-C is synthesised by type-II pneumocytes as a $21-\mathrm{kDa}$ proprotein (proSP-C), which undergoes a post-translational $\mathrm{C}$ - and N-terminal processing [28, 29]. Whilst the processing of proSP-B is completed in mvb, the final N-terminal processing step of the $\sim 6 \mathrm{kDa}$ proSP-C, processing intermediate to mature SP-C, occurs in composite and lamellar bodies [12, 29]. Despite a transport and secretion of SP-B and SP-C via the same pathway under physiological conditions, a precursor of SP-C in the intra-alveolar surfactant was detected only in one patient with PAP.

To study the intracellular distribution of SP-B and SP-C in type-II pneumocytes in normal human lungs, an immunogold triple-labelling for precursors of SP-B and SP-C, as well as mature SP-B, was performed. The current authors identified Golgi vesicles and electron-light mvb containing either precursors of SP-B or SP-C, as well as type-A (staining for precursors of SP-B and mature SP-B, but not for precursors of SP-C) and type-B (staining for precursors of SP-B and SP-C and, occasionally, mature SP-B) mvb. Previously, it was shown that the mvb were fusion products of vesicles containing either precursors of SP-C or cathepsin H [12]. Most data suggest that proSP-C is an integral membrane protein inserted into membranes in a type-II transmembrane configuration with the $\mathrm{N}$-terminus of proSP-C located in the cytoplasm [29]. The $\mathrm{N}$-terminal propeptide is necessary and sufficient for the targeting of proSP-C to postendoplasmic reticulum compartments, but not lamellar bodies, and may be involved in the fusion of vesicles containing precursors of SPC or SP-B $[29,30]$. In line with biochemical data, indicating that SP-B is necessary for the final processing and routing of precursors of SP-C to lamellar bodies [31], mvb containing only precursors of SP-C were not identified. The identification of type-A and -B mvb is also in line with data from SP-C -/mice, where a proper processing and trafficking of SP-B has been observed, as well as data from SP-B -/- mice, where an insufficient processing of SP-C has been described [32, 33].
Furthermore, in a full-term baby with a heterozygous mutation of the SP-C gene, aggregates of small vesicles containing only precursors of SP-C were detected, whilst precursors of SP-B and SP-B were normally distributed (F. Brasch, unpublished observation). Together, the biochemical and immuno-EM data provide indirect evidence that SP-B and SP-C are routed to lamellar bodies under physiological conditions, whilst under pathological conditions in idiopathic and secondary PAP, transport vesicles containing precursors of SP-B, but not SP-C, might be secreted into the alveolar space, bypassing the lamellar bodies (fig. 8).

Mature SP-C is an extremely hydrophobic, monomeric and dipalmitoylated protein with a molecular mass of $\sim 4 \mathrm{kDa}[34$, 35]. In the BAL fluid of patients with idiopathic and secondary PAP, anti-SP-C identified not only monomeric $\sim 4 \mathrm{kDa}$ SP-C, but also bands at $\sim 8 \mathrm{kDa}, \sim 12 \mathrm{kDa}$, and $\sim 16 \mathrm{kDa}$ corresponding to di- and oligomeric SP-C forms. The increase in the intensity of monomeric SP-C forms, in association with the decrease of di- and oligomeric SP-C forms under reducing conditions, is consistent with disulphide-linked di- and oligomeric SP-C forms, which are modified by partial or even complete removal of palmitate residues [36, 37]. Palmitoylation of proSP-C does not appear to be related to proprotein targeting, as substitution of the cystein residues normally undergoing palmitoylation did not influence the sorting of the protein [38]. However, dimeric SP-C might be causally related to the intra-alveolar accumulation of surfactant proteins; since it is cleared from lungs with an increased half-life, it is not removed from alveolar macrophages and it hinders clearance of SP-B and monomeric SP-C by alveolar macrophages. Furthermore, dimeric SP-C is toxic to alveolar macrophages via an increased formation of reactive oxygen species [39, 40].

In conclusion, the current authors' data show that pulmonary alveolar proteinosis is characterised not only by an intra-alveolar accumulation of surfactant protein A, B and D, but also by precursors of surfactant protein B and mono-, di- and oligomeric surfactant protein $\mathrm{C}$ forms. Furthermore, the current data provide evidence that not only an impairment of surfactant clearance by alveolar macrophages, but also 
a)

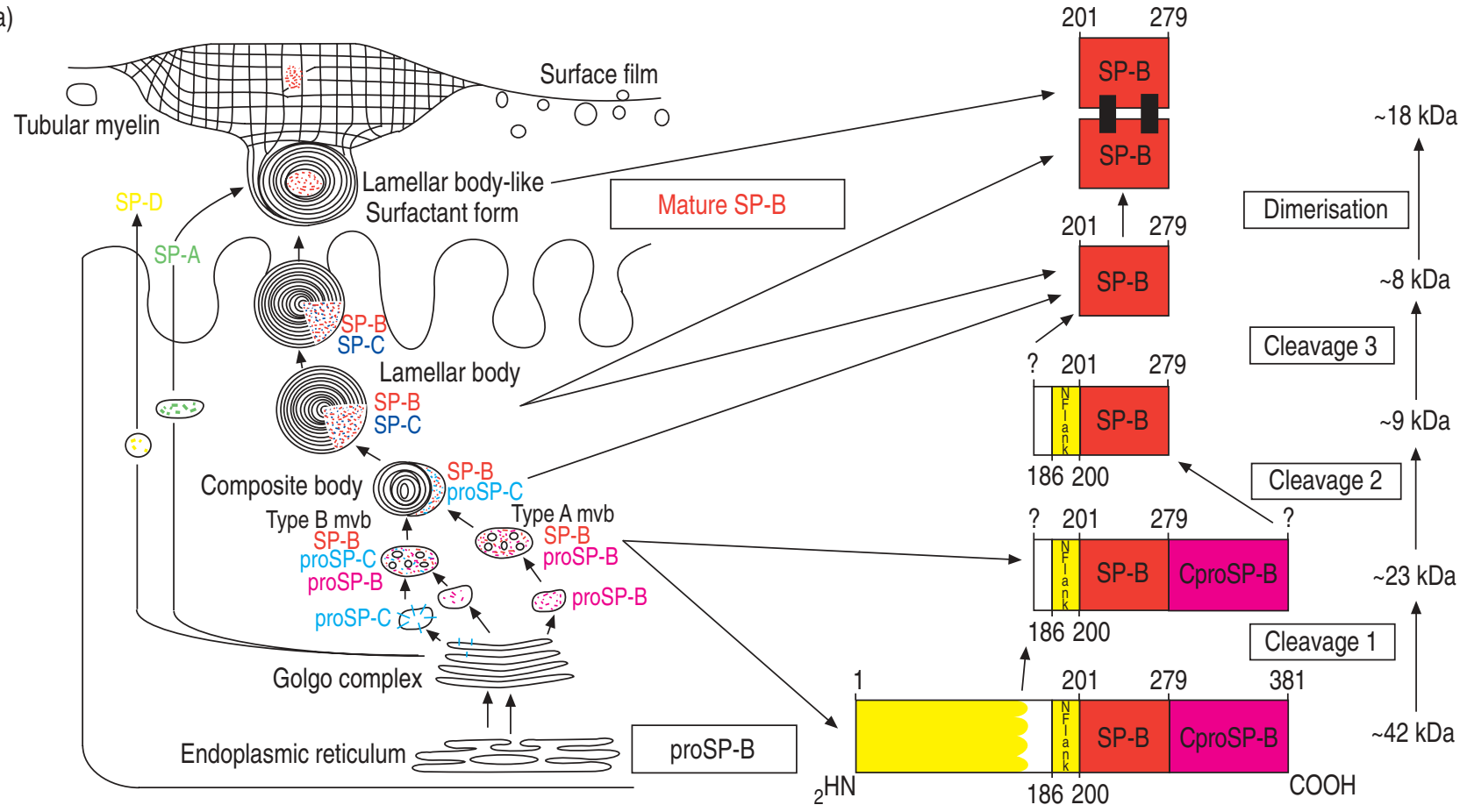

b)

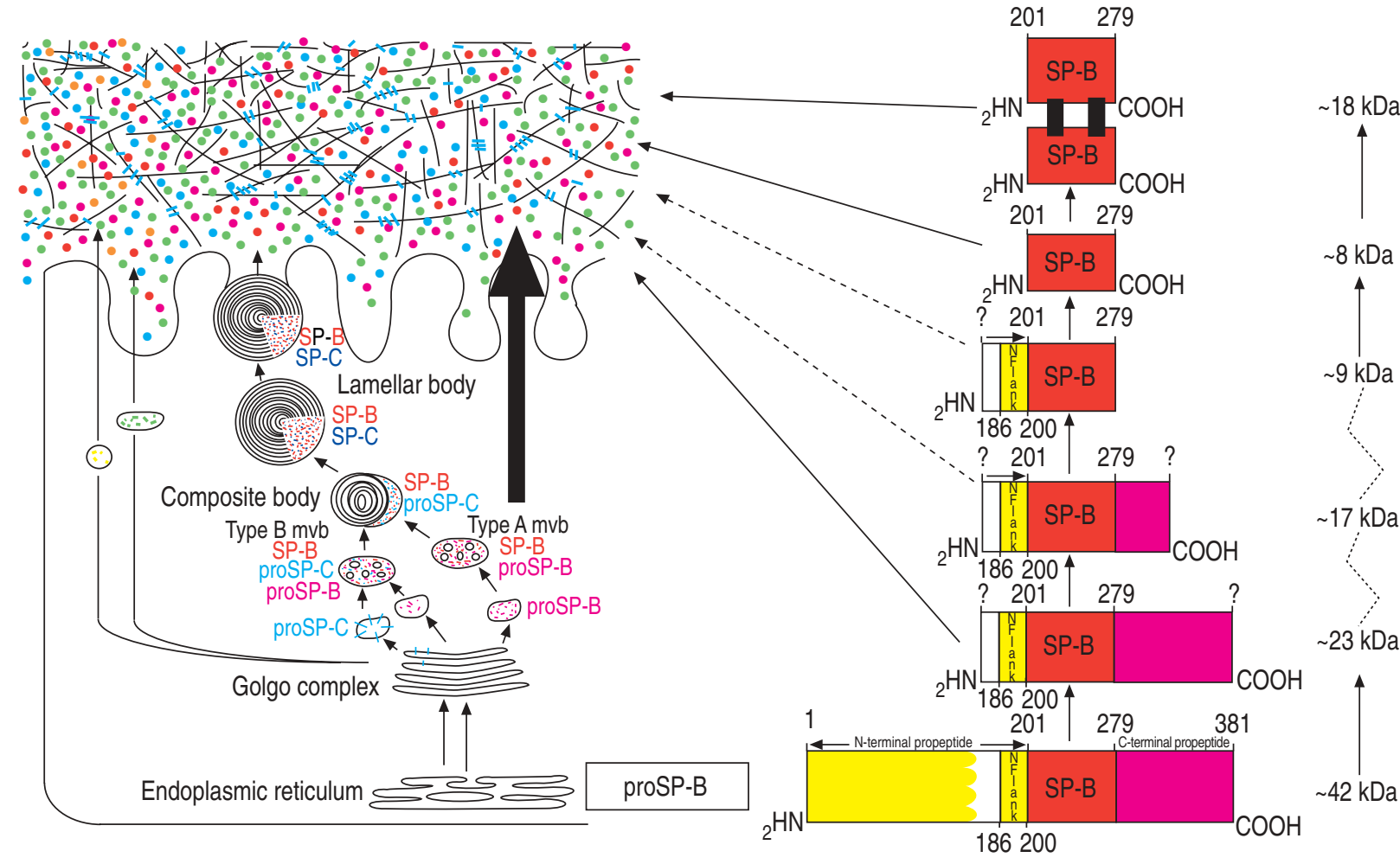

Fig. 8. - Model of intracellular trafficking of surfactant proteins (SP)-A, B, C and D, as well as processing of proSP-B in type-II pneumocytes of normal human lungs and patients with pulmonary alveolar proteinosis (PAP). In normal human lungs (a), SP-B and SP-C are synthesised in the endoplasmic reticulum and transported via Golgi vesicles and multivesicular bodies (mvb) to lamellar bodies. Immunogold labelling distinguished transport vesicles containing either precursor of SP-B or SP-C and indicated that the type-B mvb are fusion products of these vesicles. Mature SP-C and SP-B are secreted via the lamellar bodies in the intra-alveolar space under physiological conditions [12, 14, 26], whereas SP-A and SP$\mathrm{D}$ largely bypass the lamellar bodies [13, 27, 41]. The processing of proSP-B was adapted from BRASCH et al. [14]. In patients with PAP (b), SP-A and SP-D and, in addition, mature SP-C and SP-B are secreted in the intra-alveolar space via small transport vesicles and lamellar bodies, respectively. An abnormal secretion of transport vesicles containing precursors of SP-B leads to an intra-alveolar accumulation of processing intermediates of proSP-B.

an abnormal secretion of transport vesicles containing precursors of surfactant protein B (but not C) and an insufficient palmitoylation of surfactant protein $\mathrm{C}$, which may lead to the formation of di- and oligomeric surfactant protein $\mathrm{C}$ forms, play a role in the pathogenesis of pulmonary alveolar proteinosis. 


\begin{abstract}
Acknowledgements. The authors would like to thank K. Nakata for the analysis of pretreatment sera of five patients for GM-CSF-neutralising autoantibodies, and G. Goeckenjan, W. Hartmann, P. Heitz, E. Kaukel, D. Köhler, U. Schmid, G. Schultze-Werninghaus, H. Steppling, H-N. Macha, J. Altenwerth, C. Eschenbruch, H. Heyenga, W. Jochum, R. Kappes, C. Oehlschlegel, J. Schildge, E. Scholtze and P. Wex for the referral of lung biopsies and BAL fluid from patients with PAP. The excellent technical assistance of S. Geiger, M. Kochem and U. Thomek is highly appreciated.
\end{abstract}

\section{References}

1. Rosen SH, Castleman B, Liebow AA. Pulmonary alveolar proteinosis. N Engl J Med 1958; 258: 1123-1142.

2. Bonfield TL, Russell D, Burgess S, Malur A, Kavuru MS, Thomassen MJ. Autoantibodies against granulocyte-macrophage colony-stimulating factor are diagnostic for pulmonary alveolar proteinosis. Am J Respir Cell Mol Biol 2002; 27: 481-486.

3. Kitamura T, Tanaka N, Watanabe J, et al. Idiopathic pulmonary alveolar proteinosis as an autoimmune disease with neutralizing antibody against granulocyte-macrophage colony-stimulating factor. J Exp Med 1999; 190: 875-880.

4. Reed JA, Ikegami M, Cianciolo ER, et al. Aerosolized GMCSF ameliorates pulmonary alveolar proteinosis in GMCSF-deficient mice. Am J Physiol 1999; 276: L556-L563.

5. Seymour JF, Presneill JJ, Schoch OD, et al. Therapeutic efficacy of granulocyte-macrophage colony-stimulating factor in patients with idiopathic acquired alveolar proteinosis. Am J Respir Crit Care Med 2001; 163: 524-531.

6. Seymour JF, Presneill JJ. Pulmonary alveolar proteinosis: progress in the first 44 years. Am $J$ Respir Crit Care Med 2002; 166: 215-235.

7. Wang BM, Stern EJ, Schmidt RA, Pierson DJ. Diagnosing pulmonary alveolar proteinosis. A review and an update. Chest 1997; 111: 460-466.

8. Martin RJ, Coalson JJ, Rogers RM, Horton FO, Manous LE. Pulmonary alveolar proteinosis: the diagnosis by segmental lavage. Am Rev Respir Dis 1980; 121: 819-825.

9. Gonzales LW, Angampalli S, Guttentag SH, et al. Maintenance of differentiated function of the surfactant system in human fetal lung type II epithelial cells cultured on plastic. Pediatr Pathol Mol Med 2001; 20: 387-412.

10. Solarin KO, Ballard PL, Guttentag SH, Lomax CA, Beers MF. Expression and glucocorticoid regulation of surfactant protein C in human fetal lung. Pediatr Res 1997; 42: 356364.

11. Korimilli A, Gonzales LW, Guttentag SH. Intracellular localization of processing events in human surfactant protein B biosynthesis. J Biol Chem 2000; 275: 8672-8679.

12. Brasch F, Ten Brinke A, Johnen G, et al. Involvement of cathepsin $\mathrm{H}$ in the processing of the hydrophobic surfactantassociated protein $\mathrm{C}$ in type II pneumocytes. Am J Respir Cell Mol Biol 2002; 26: 659-670.

13. Ochs M, Johnen G, Müller KM, et al. Intracellular and intraalveolar localization of surfactant protein A (SP-A) in the parenchymal region of the human lung. Am J Respir Cell Mol Biol 2002; 26: 91-98.

14. Brasch F, Johnen G, Winn-Brasch A, et al. Surfactant protein B in type II pneumocytes and intra-alveolar surfactant forms of human lungs. Am J Respir Cell Mol Biol 2004; 30: 449-458.

15. Reed JA, Ikegami M, Robb L, Begley CG, Ross G, Whitsett JA. Distinct changes in pulmonary surfactant homeostasis in common beta-chain- and GM-CSF-deficient mice. Am J Physiol Lung Cell Mol Physiol 2000; 278: L1164-L1171.

16. Hook GE. Alveolar proteinosis and phospholipidoses of the lungs. Toxicol Pathol 1991; 19: 482-513.
17. Huffman JA, Hull WM, Dranoff G, Mulligan RC, Whitsett JA. Pulmonary epithelial cell expression of GM-CSF corrects the alveolar proteinosis in GM-CSF-deficient mice. J Clin Invest 1996; 97: 649-655.

18. Nishinakamura R, Wiler R, Dirksen U, et al. The pulmonary alveolar proteinosis in granulocyte-macrophage colonystimulating factor/interleukins $3 / 5$ beta $\mathrm{c}$ receptor-deficient mice is reversed by bone marrow transplantation. J Exp Med 1996; 183: 2657-2662.

19. Robb L, Drinkwater CC, Metcalf D, et al. Hematopoietic and lung abnormalities in mice with a null mutation of the common beta subunit of the receptors for granulocytemacrophage colony-stimulating factor and interleukins 3 and 5. Proc Natl Acad Sci USA 1995; 92: 9565-9569.

20. Doyle IR, Davidson KG, Barr HA, Nicholas TE, Payne K, Pfitzner J. Quantity and structure of surfactant proteins vary among patients with alveolar proteinosis. Am J Respir Crit Care Med 1998; 157: 658-664.

21. Crouch E, Persson A, Chang D. Accumulation of surfactant protein $\mathrm{D}$ in human pulmonary alveolar proteinosis. $\mathrm{Am}$ J Pathol 1993; 142: 241-248.

22. Alberti A, Luisetti M, Braschi A, et al. Bronchoalveolar lavage fluid composition in alveolar proteinosis. Early changes after therapeutic lavage. Am J Respir Crit Care Med 1996; 154: 817-820.

23. Honda Y, Takahashi H, Shijubo N, Kuroki Y, Akino T. Surfactant protein A concentration in bronchoalveolar lavage fluids of patients with pulmonary alveolar proteinosis. Chest 1993; 103: 496-499.

24. Voorhout WF, Veenendaal T, Haagsman HP, et al. Intracellular processing of pulmonary surfactant protein B in an endosoma1/lysosomal compartment. Am J Physiol 1992; 263: L479-L486.

25. Phelps DS, Floros J. Localization of surfactant protein synthesis in human lung by in situ hybridization. Am Rev Respir Dis 1988; 137: 939-942.

26. Weaver TE. Synthesis, processing and secretion of surfactant proteins B and C. Biochim Biophys Acta 1998; 1408: 173-179.

27. Gobran LI, Rooney SA. Regulation of SP-B and SP-C secretion in rat type II cells in primary culture. Am J Physiol Lung Cell Mol Physiol 2001; 281: L1413-L1419.

28. Vorbroker DK, Voorhout WF, Weaver TE, Whitsett JA. Post-translational processing of surfactant protein $\mathrm{C}$ in rat type II cells. Am J Physiol 1995; 269: L727-L733.

29. Johnson AL, Braidotti P, Pietra GG, et al. Post-translational processing of surfactant protein-C proprotein: targeting motifs in the $\mathrm{NH}(2)$-terminal flanking domain are cleaved in late compartments. Am J Respir Cell Mol Biol 2001; 24: 253-263.

30. Conkright JJ, Bridges JP, Na CL, et al. Secretion of surfactant protein $\mathrm{C}$, an integral membrane protein, requires the N-terminal propeptide. J Biol Chem 2001; 276: 1465814664.

31. Lin S, Akinbi HT, Breslin JS, Weaver TE. Structural requirements for targeting of surfactant protein B (SP-B) to secretory granules in vitro and in vivo. J Biol Chem 1996; 271: 19689-19695.

32. Glasser SW, Burhans MS, Korfhagen TR, et al. Altered stability of pulmonary surfactant in SP-C-deficient mice. Proc Natl Acad Sci USA 2001; 98: 6366-6371.

33. Clark JC, Wert SE, Bachurski CJ, et al. Targeted disruption of the surfactant protein B gene disrupts surfactant homeostasis, causing respiratory failure in newborn mice. Proc Natl Acad Sci USA 1995; 92: 7794-7798.

34. Johansson J. Structure and properties of surfactant protein C. Biochim Biophys Acta 1998; 1408: 161-172.

35. Stults JT, Griffin PR, Lesikar DD, Naidu A, Moffat B, Benson BJ. Lung surfactant protein SP-C from human, bovine, and canine sources contains palmityl cysteine thioester linkages. Am J Physiol 1991; 261: L118-L125.

36. Voss T, Schafer KP, Nielsen PF, et al. Primary structure differences of human surfactant-associated proteins isolated 
from normal and proteinosis lung. Biochim Biophys Acta 1992; 1138: 261-267.

37. Gustafsson M, Griffiths WJ, Furusjo E, Johansson J. The palmitoyl groups of lung surfactant protein $\mathrm{C}$ reduce unfolding into a fibrillogenic intermediate. J Mol Biol 2001; 310: 937-950.

38. Ten Brinke A, Batenburg JJ, Gadella BM, Haagsman HP, Vaandrager AB, van Golde LM. The juxtamembrane lysine and arginine residues of surfactant protein $\mathrm{C}$ precursor influence palmitoylation via effects on trafficking. $\mathrm{Am}$ J Respir Cell Mol Biol 2001; 25: 156-163.
39. Li ZY, Suzuki Y, Kurozumi M, Shen HQ, Duan CX Removal of a dimeric form of surfactant protein $\mathrm{C}$ from mouse lungs: its acceleration by reduction. J Appl Physiol 1998; 84: 471-478.

40. Shen HQ, Duan CX, Li ZY, Suzuki Y. Effects of proteinosis surfactant proteins on the viability of rat alveolar macrophages. Am J Respir Crit Care Med 1997; 156: 16791687.

41. Osanai K, Mason RJ, Voelker DR. Trafficking of newly synthesized surfactant protein A in isolated rat alveolar type II cells. Am J Respir Cell Mol Biol 1998; 19: 929-935. 